\title{
De la lucha al testimonio. Las representaciones audiovisuales e interactivas del cáncer en los videojuegos
}

\author{
From fight to testimony. Audiovisual and interactive representations of \\ cancer in video games (abstract: p. 16)
}

Da luta ao testemunho. Representações audiovisuais e interativas do câncer em videogames (resumo: p. 16)

\author{
Salvador Gómez-García ${ }^{(a)}$ \\ <salvadorgomez@hmca.uva.es> \\ Nuria Navarro-Sierra(b) \\ <nuria.navarro.sierra@urjc.es> \\ José-Agustín Carrillo-Vera(c) \\ <joseagustin.carrillo@um.es> iD
}

\author{
(a) Departamento de Periodismo, \\ Facultad de Filosofía y Letras \\ Universidad de Valladolid. Plaza del \\ Campus, S/N, Valladolid, España, \\ 47011. \\ (b) Departamento de Ciencias de la \\ Comunicación y Sociología, Facultad \\ de Ciencias de la Comunicación, \\ Universidad Rey Juan Carlos. Madrid, \\ España. \\ (c) Departamento de Información \\ y Documentación, Facultad de \\ Comunicación y Documentación \\ Universidad de Murcia. Murcia, \\ España.
}

Esta investigación explora la intersección entre el cáncer - una de las principales causas de mortalidad de las sociedades contemporáneas - y uno de los medios más significativos de la cultura popular en el siglo XXI: los videojuegos. El análisis de contenido de los mensajes ha comprendido ocho videojuegos que, durante los últimos 15 años, revelan un planteamiento narrativo en torno a la afección, pacientes, tratamientos y desenlace de la enfermedad. Los resultados señalan una construcción en la que perviven sus rasgos más populares dirigidos al empoderamiento social frente a la enfermedad a través del recurso a la ciencia, el respaldo social o las convicciones espirituales.

Palabras clave: Cáncer. Videojuego. Juegos serios. Industrias culturales. Información y concienciación. 


\section{Introducción}

El cáncer es una de las principales causas de mortalidad en las sociedades desarrolladas destacando por su elevada prevalencia, incidencia en ambos géneros y tasa de deceso ${ }^{1}$. La WHO cifró 8,8 millones de defunciones en 2015 subrayando los tipos más frecuentes de esta enfermedad - pulmonar, hepático, colorrectal, gástrico y mamario- y sus principales causas de riesgo: índice de masa corporal elevado, ingesta reducida de frutas y verduras, falta de actividad física, consumo de tabaco y de alcohol ${ }^{2}$.

El trágico rol de esta enfermedad explica su constante presencia en los medios de comunicación tradicionales, que suelen centrarse en las noticias e informaciones sobre factores de riesgo y su prevención ${ }^{3}$ con una tendencia a la sobrerrepresentación informativa ${ }^{4}$. Este foco de atención sobre la enfermedad se refleja en medios populares como el cine, la televisión o el cómic ${ }^{(\mathrm{d})}$, que han ofrecido visiones adaptadas a sus públicos y sus rasgos discursivos. Su presencia en la cultura popular se ha ampliado en el contexto de los nuevos medios a través de, por ejemplo, comunidades virtuales ${ }^{8}$, redes sociales como Facebook ${ }^{9}$ o narrativas difundidas a través de Youtube ${ }^{10}$.

Esta participación en el imaginario social ${ }^{(\mathrm{e})}$ sobre la enfermedad a través de los medios imbrica un amplio conjunto de temáticas y planteamientos. Una propuesta no exhaustiva de los mismos abarca diferentes cuestiones. La primera de ellas aborda la divulgación de las estrategias de prevención a dos niveles: primario (a través de hábitos saludables) y secundario (relacionado con la detección temprana). Unas fórmulas que no han logrado su máxima efectividad puesto que su incentivación reduciría la mortalidad del cáncer en un $70 \%{ }^{14}$ asumiendo que los medios de comunicación juegan un papel fundamental en este proceso ${ }^{15}$.

Un segundo aspecto de interés analiza el proceso de empoderamiento a través de un amplio conjunto de estrategias entre las que destaca el pensamiento positivo impulsado por los vínculos familiares ${ }^{16}$ y el uso de los medios de comunicación ${ }^{17}$. Entre ellos, la postura ante la enfermedad como una "guerra contra el cáncer" ${ }^{18,19}$ es su metáfora más recurrente de los medios de comunicación sobre esta enfermedad ${ }^{20}$. La doble lectura de este principio de empoderamiento señala, por un lado, las bondades de esta formulación -comprensión de la enfermedad y empoderamiento- pero, por otro lado, sus principales contrapartidas -responsabilidad excesiva del paciente sobre la eficacia del tratamiento, frustración, detrimento de las conductas preventivas o simplificación de la percepción sobre la enfermedad ${ }^{21,22}$ - También se ha identificado un interés por identificar el tipo de relaciones que se establece entre los actores (pacientes, familiares, profesionales sanitarios, entorno, etc.), sus flujos comunicativos y las consecuencias de la enfermedad sobre dichos vínculos planteando una dimensión social que ha sido abordado desde un conjunto de perspectivas tan amplias como el fenómeno ${ }^{23}$.

Por último, la interpretación de la enfermedad y la muerte en las sociedades contemporáneas se combina con el trágico rol de esta enfermedad genética que ha implicado nuevas formas de tratamiento en la medicina moderna ${ }^{24}$. En ese sentido, destaca la confianza en la medicina, pero también las propias convicciones religiosas del paciente como forma de afrontar la enfermedad con éxito o desde una perspectiva paliativa ${ }^{25}$.

El objetivo de esta investigación se sitúa en sintonía con estas lecturas para analizar las manifestaciones de una enfermedad como el cáncer a través del estudio cualitativo de uno de los medios de comunicación más relevantes del siglo XXI: los videojuegos (d) La relación entre la enfermedad y su plasmación audiovisual (cine y televisión) ha estado muy presente reflejando ámbitos muy diversos: aprendizaje de actitudes, patologías, relaciones entre pacientes y profesionales, diferencias socioculturales y un amplio etcétera ${ }^{5}$. De forma similar, los cómics han abordado la enfermedad integrándola como un elemento que interviene en el relato ${ }^{6}$ y como una construcción testimonial a través de discursos introspectivos y en primera persona?.

(e) A pesar de la cierta ambigüedad y multiplicidad de interpretaciones y definiciones a las que da lugar el concepto de imaginario social ${ }^{11}$, en este trabajo hacemos referencia a una de sus concepciones más canónicas, la que lo establece como creación de concepciones imaginarias sociales ${ }^{12} \mathrm{O}$ esquemas socialmente construidos para tratar de explicar una realidad y contribuir a interpretarla ${ }^{13}$. 
y su distribución a través de internet ${ }^{26}$. Dicha propuesta se traduce en una pregunta de investigación que se interroga sobre cuáles son las contribuciones al imaginario social que -desde el ámbito de los videojuegos- se han desarrollado en torno a esta enfermedad.

\section{Método}

La muestra de esta investigación comprende aquellos juegos cuyos elementos narrativos están vinculados de forma directa al cáncer como enfermedad. La localización de la muestra se realizó a través de búsquedas empleando términos clave (cáncer y videojuegos) en las principales plataformas de distribución digital de juegosSteam, Apple Store y Google Play-, bases de datos -Moby Dick, EuroGamer y Serious Games Database - y bases de datos académicas - WoK, Scopus y Google Scholar. El criterio de selección fue una alusión explícita al cáncer en la descripción del producto y que se presentase como un juego. Se descartaron aquellos que, aunque hacen referencia al cáncer, no abordan un proceso de construcción simbólico de la enfermedad. Es el caso del advergame Rooftop Runner (Cancer Research UK \& Matmi Games, 2009), juego de carreras sobre tejados enmarcado en una campaña viral para obtener fondos contra el cáncer ${ }^{27}$. Tampoco se han incluido Help Fight Breast Cancer (Silver Dollar Games, 2011), un cuestionario sobre prevención sin abordar una construcción narrativa, o proyectos colaborativos de ciencia ciudadana como Play to cure: Genes in space (Scientists at Cancer Research UK, 2014) o Reverse the odds (Cancer Research UK, 2016).

Por tanto, la muestra final, que comprende ocho resultados, presenta un criterio de conexión entre el núcleo narrativo, representación audiovisual y los aspectos interactivos del juego, en el marco de los parámetros del diseño consciente de los valores transmitidos por el videojuego ${ }^{28}$. El esquema de análisis de los videojuegos propuestos parte de su contribución al imaginario social y su influencia cultural en las sociedades contemporáneas ${ }^{29}$, y de diferentes modelos de análisis narrativo ${ }^{30-32}$ que se han conectado con las construcciones culturales sobre el cáncer ${ }^{18,33}$. Por tanto, el análisis se configura en torno a una serie de elementos centrales (la enfermedad, los pacientes, los tratamientos y las formas de combatir la afección y el tipo de desenlace que se plantea) en su relación a las características discursivas del videojuego (Figura 1).

\section{Aproximación narrativa-audiovisual de la muestra}

El planteamiento narrativo, estético, lúdico y tecnológico de los juegos analizados está en correspondencia con su evolución (2003-2018). A continuación, se presentan las premisas básicas de cada uno de ellos:

Ben's Game (Make-A-Wish, 2003). Un niño es miniaturizado en su propio cuerpo junto a su monopatín con el objetivo de destruir células cancerígenas y obtener siete escudos que le protegerán contra los efectos secundarios de la quimioterapia. Esta propuesta de la fundación Make- $A$-Wish facilitó que Ben Duskin, un niño de nueve años enfermo de leucemia, colaborara con Lucas Arts para diseñar un juego que ofreciese su experiencia y se difundiese en hospitales para mejorar la motivación de los pacientes más jóvenes ${ }^{34}$. 

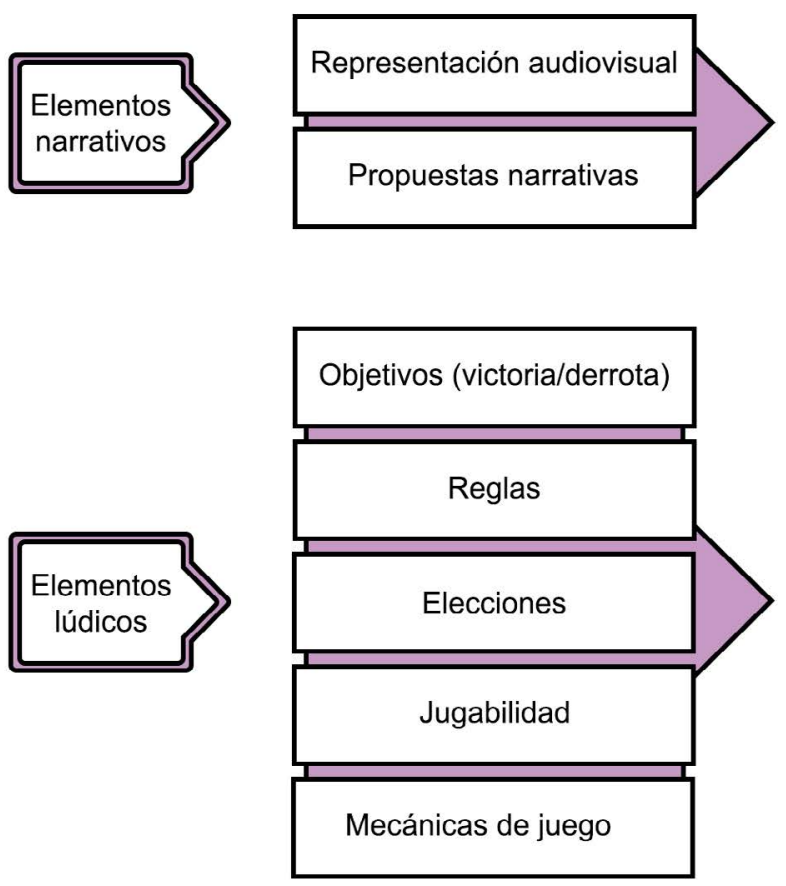

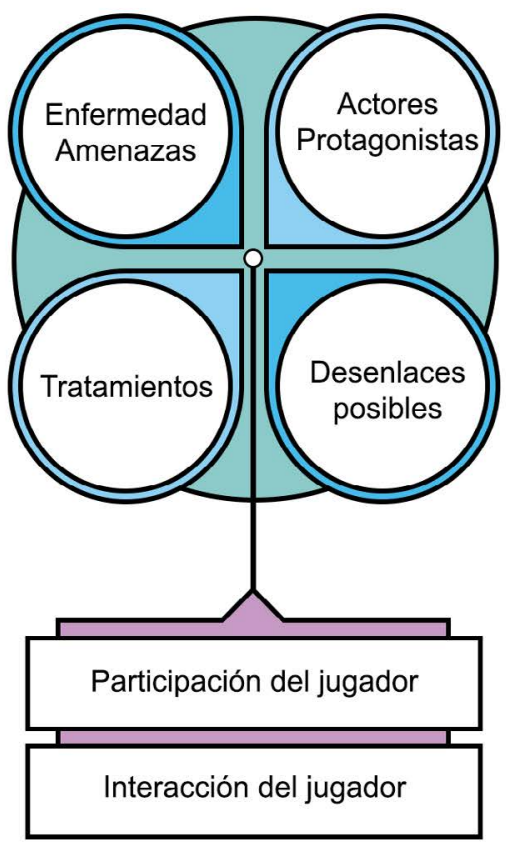

Interacción del jugador

Figura 1. Modelo de análisis.

Re-Mission (Hope Lab, 2006). El jugador encarna a un nanobot, Roxxi, diseñado para combatir el cáncer y las infecciones a nivel celular tras ser introducido en el cuerpo humano. A lo largo de una serie de misiones, el jugador tendrá que ayudar a adolescentes enfermos de cáncer a superar la enfermedad. Este juego también se empleó en contextos sanitarios para analizar sus efectos en la conducta de los pacientes ${ }^{35}$.

Cancer Game (Wei-Wei, 2007). Una aventura gráfica que plantea un recorrido a través de diferentes partes del cuerpo humano -pulmones, hígado, estomago e intestinos-. Cada uno de estos órganos se asocia a una pantalla-puzle cuya resolución ayuda a comprender los hábitos perniciosos -tabaco, alcohol, ultraprocesados, etc.asociados a esta enfermedad ${ }^{36}$.

Re-Mission 2 (Hope Lab, 2013). Colección de mini-juegos, con una premisa narrativa simplificada respecto a su antecesor, en la que los jugadores encarnan a nanobots que combaten al cáncer ayudados por el sistema inmunitario, antibióticos y quimioterapia.

NanoDoc (MIT, 2013). Juego de estrategia online desarrollado por el Laboratory of Multiscale Regenerative Technologies bajo la etiqueta de crowdsourcing nanomedicine. Su objetivo es buscar patrones que ayuden a eliminar tumores gracias a la nanotecnología ${ }^{37}$.

AlphaBeatCancer (Bababa, 2016). Sencillo juego para dispositivos móviles dirigido a un público infantil en el que un oso de peluche se enfrenta a diversos mini-juegos relacionados con un tratamiento contra el cáncer (extracción de sangre, medicación, nutrición, etc.). 
That Dragon, Cancer (Numinous Game, 2016). El juego más popular y galardonado de la muestra. Un proyecto híbrido entre documental cinematográfico y aventura gráfica desarrollado por Ryan Green en el que aborda su experiencia personal como padre de Joel, un niño de 5 años afectado por una variedad extraña y muy agresiva de cáncer. El jugador participa en un recorrido por la experiencia de esta familia en la lucha contra la enfermedad a lo largo de las catorce escenas en las que se divide el juego.

I, Hope (Kenny Roy, 2018). La aldea de Hope es atacada por un monstruo llamado Cáncer. Esta joven muchacha, guiada por su abuelo, deberá buscar las armas y el conocimiento que necesita para poder vencer al monstruo y devolver la paz a su hogar.

Esta aproximación ofrece rasgos de interés que son comunes en la muestra. El primero de ellos es cómo se aborda la experiencia del usuario. Todos son juegos de corta duración o con capacidad de fragmentar el tiempo de juego. Por tanto, son productos orientados tanto a jugadores tradicionales como casuales de cara a facilitar la difusión del discurso en un amplio rango de públicos. Otro aspecto de interés es el género en el que se inscriben estos juegos y que condiciona su discurso y sus mecánicas. De forma concreta, predominan aquellos más populares entre los jóvenes -acción y aventura - diferenciados en su jugabilidad: shoot 'em up, aventura gráfica o plataformas ${ }^{(\mathrm{f})}$. Además, la estética animada y las premisas narrativas también son acordes a un público juvenil.

\section{Resultados}

\section{Los diferentes rostros de la enfermedad}

La representación del cáncer como una amenaza tiene diferentes grados de abstracción que se analizan a continuación:

Cuando el cáncer es mostrado como una amenaza única tangible -el modo de representación más simplificado- es caracterizado como un monstruo o un ser grotesco cuya vileza se ve dibujada en su propia imagen, que pasa del propio concepto del cáncer en abstracto a la imagen física de un monstruo (I, hope) o a manifestaciones concretas como el cáncer de hígado o pulmón (Cancer Game) (Figura 2).

Este tipo de construcción está asociada a juegos que no plantean respuestas específicas sobre los motivos de la afección o su evolución, sino que presentan una situación evitable, ya sea a través de hábitos de vida saludables (Cancer Game) o de infundir un mensaje de empoderamiento de los más jóvenes frente a la enfermedad ( $I$, Hope).

El cáncer como un conjunto de amenazas múltiples tangibles se asocia a elementos propios de la enfermedad desde el punto de vista médico. Por un lado, se plantea una representación abstracta asociada a algún aspecto de la enfermedad o de su tratamiento, como en Ben's Game, donde los efectos secundarios de la quimioterapia -escalofríos, vómitos, varicela (chickenpox en inglés), fiebre, sangrados, pérdida de cabello o erupciones- toman la forma de los enemigos que hay que derrotar -el hombre de hielo, monstruo de vómito, un pollo gigante, una bola gigante de fuego, un vampiro, una Qball y un tornado- (Figura 3). (f) Se ha seguido una taxonomía que centrada en sus rasgos básicos ${ }^{38}$, identificando como shoot 'em up (o shooters) a los juegos que "implican disparar, y a menudo destruir, a oponentes u objetos"38; como aventuras gráficas a los "situados en un 'mundo' formado por múltiples habitaciones/ pantallas conectadas y cuyo objetivo [...] debe ser resuelto tras varios pasos, por ejemplo, encontrando unas llaves y abriendo puertas en otras áreas"38; y plataformas a los que "avanzan por una serie de niveles que se superan corriendo, escalando, saltando y por otros medios de locomoción. También implican evitar objetos que caen o el conflicto con personajes controlados por el ordenador"38. 

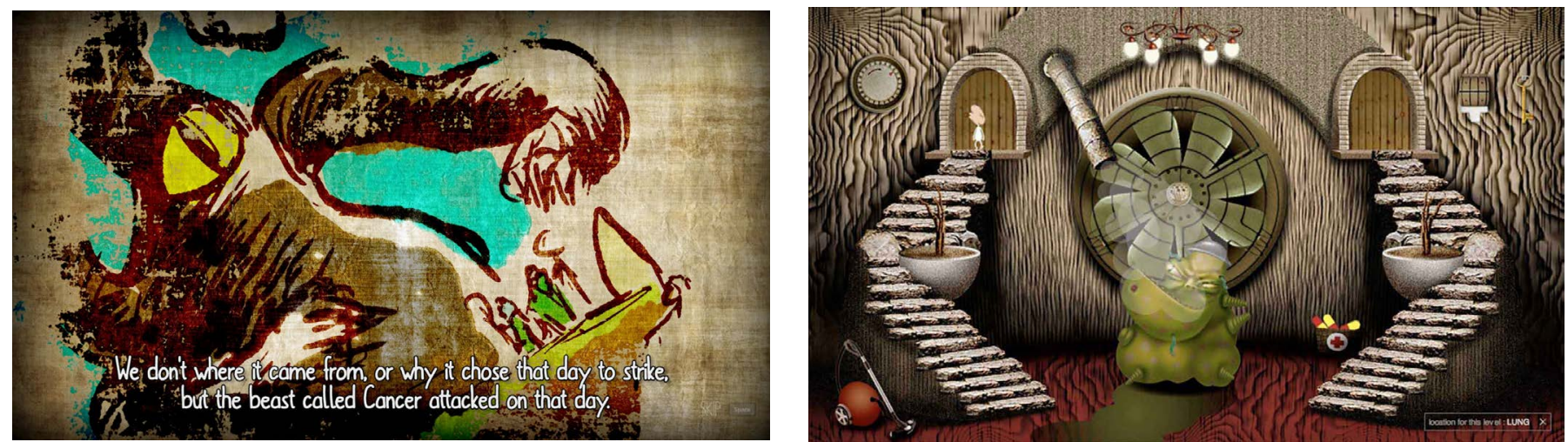

Figura 2. El cáncer como amenaza única tangible
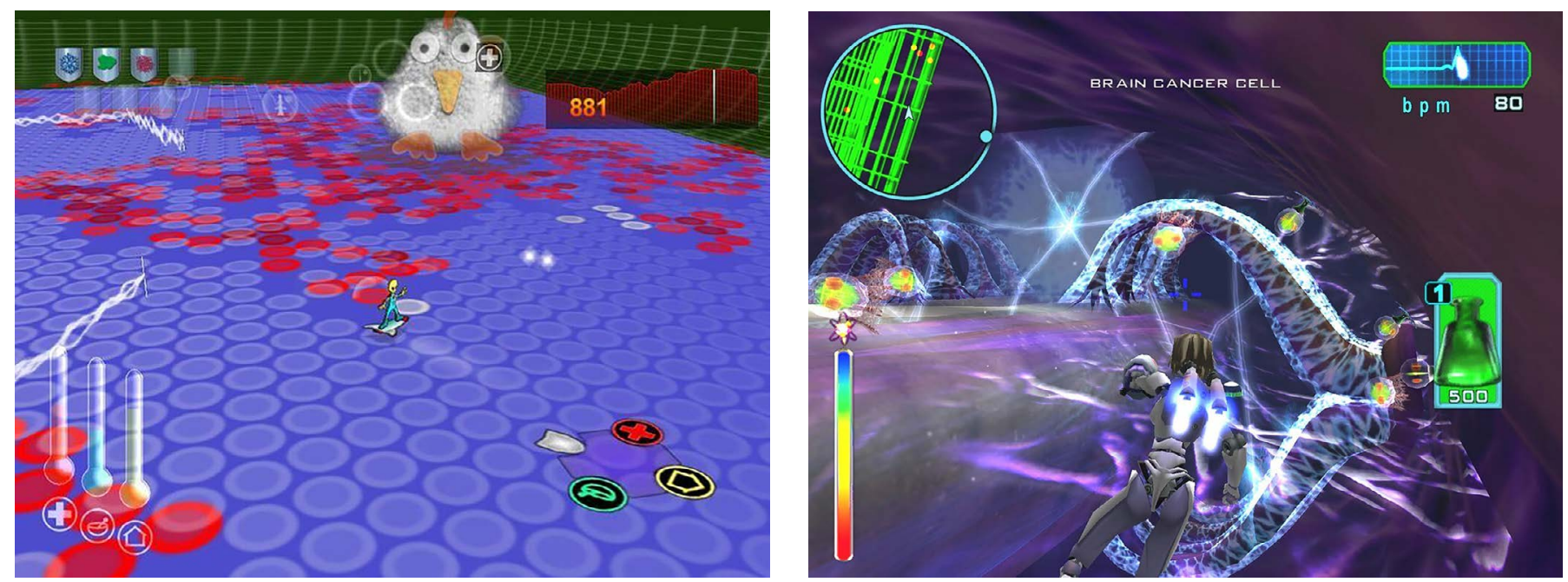

Figura 3. El cáncer como amenaza múltiple

Por otro lado, Re-Mission y Re-Mission 2 optan por una representación científica mostrando tanto bacterias como células cancerígenas: leucemia, linfomas u osteosarcoma entre otras. Esa elección en el diseño proviene de los objetivos terapéuticos y educativos del propio juego para ayudar a comprender la enfermedad. Se facilita, de esta forma, presentar los rasgos propios del discurso científico asociado a la enfermedad junto a las fórmulas para combatirla.

Por último, That Dragon, Cancer articula una relación con la enfermedad en la que ésta nunca adquiere una formulación concreta o tangible. En otras palabras, la enfermedad no presenta una concreción visual, pero sí sus efectos sobre la familia Green; el matrimonio (Ryan y Amy) y sus cuatro hijos, de los cuales Joel, de 5 años, padece un raro tipo de cáncer que es extremadamente agresivo. Las doce escenas que componen el juego presentan aspectos cotidianos de la familia Green en su convivencia con la enfermedad de Joel entremezclados con escenarios oníricos que reflejan deseos, pensamientos o angustias interiores (Figura 4). 

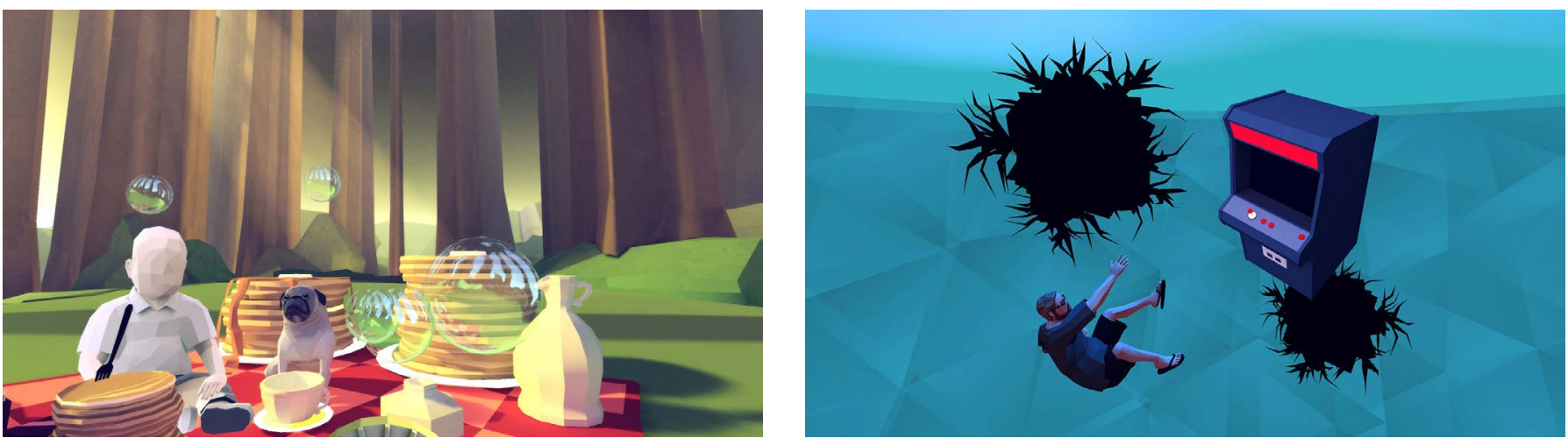

Figura 4. El cáncer como presencia intangible

La ambigüedad que refleja That Dragon, Cancer en su representación de la enfermedad se advierte en la ausencia de objetivos concretos o de una condición de victoria. Este hecho se debe a su concepción como testimonio de la familia Green -y en línea con lecturas actuales sobre la enfermedad que desestima su planteamiento en clave de conflicto ${ }^{39}$-. De esta forma, se elimina el interés de obtener unos resultados óptimos a favor de establecer una conexión con la familia de Joel durante el juego tal y como planteaba su padre "[...] gran parte de la vida no se trata de saber las respuestas, sino de caminar unos junto a otros desconociéndolas" ${ }^{30}$.

Las formulaciones anteriores reflejan la pervivencia de una construcción simbólica sobre la enfermedad "como una maldición; metafóricamente, era el bárbaro dentro del cuerpo" ${ }^{18}$. Un hecho que se refleja tanto en que la personalización del cáncer condiciona al resto de elementos del discurso -formas de afrontar la afección, desenlace, etc.- como en la capacidad de concretar sus efectos.

\section{Nosotros en la pantalla: jugadores, espectadores y pacientes}

La mayoría de protagonistas de los videojuegos de la muestra son figuras juveniles relacionados con personajes emblemáticos de otros videojuegos. Por ejemplo, Roxxi (Re-Mission) es una joven nanobot que se introduce en el plasma sanguíneo para combatir la enfermedad y que se asemeja a Lara Croft, la protagonista de la conocida saga Tomb Raider, la estética de oso de peluche de Alpha (AlphaBeatCancer); Hope (I, Hope) es una adolescente guerrera con reminiscencias a Link, el protagonista de Zelda, una de las sagas más famosas de Nintendo; o, por último, Ben, en su monopatín cohete (Ben's Game), se inspira en la popular franquicia de videojuegos deportivos Tony Hawk's. La apuesta por unos avatares fácilmente identificables en las referencias populares del mundo de los videojuegos influye en su aceptación tanto por aquellos familiarizados con esta cultura como por parte de un público juvenil.

That Dragon, Cancer y Cancer Game emplean una lógica diferente. En el primero, los padres de Joel articulan la narración para ofrecer el testimonio de aquellos que acompañan al enfermo. Por otro lado, Cancer Game emplea un personaje anónimo que recorre diferentes órganos del cuerpo humano haciendo frente a las amenazas contra su salud. El ser el único orientado a las labores de prevención responde, en 
palabras de su creador, a una apuesta "por presentar una forma lúdica de involucrar a las personas en la exploración de síntomas y causas"36.

La construcción de protagonistas se complementa con la de otros actores que participan en la narración. Su presencia se resume en tres grandes modelos: guías morales, autoridades médicas y elementos del cuerpo humano.

El primero se refleja en el abuelo de Hope, que encomienda a su nieta la misión de obtener armas y conocimiento para hacer frente a la enfermedad. Se ubica de esta manera un integrante de la esfera familiar que orienta los esfuerzos y objetivos del jugador.

La autoridad médica se representa tanto como una fuente de información honesta pero inflexible (That Dragon, Cancer) o como artífice de la ayuda que recibe el cuerpo humano para eliminar la enfermedad (Re-Mission o AlphaBeatCancer). En este caso, la representación del efecto de los tratamientos plantea un mensaje conductual para "garantizar el seguimiento de los pacientes del tratamiento farmacológico, uno de los principales problemas de la medicina actual" 39 .

Por último, algunos juegos presentan diferentes elementos del cuerpo humano: tanto órganos como elementos propios del torrente sanguíneo - plaquetas, glóbulos rojos, etc.-. Su rol se centra en ser objetivos del cáncer, campos de batalla (en el caso de los órganos) o testigos de los esfuerzos del jugador por eliminar la amenaza.

\section{Jugar es vencer: mecánicas y acciones}

Las propuestas argumentativas en relación con los personajes se completan con los elementos lúdicos que constituyen la naturaleza del juego. Dichas mecánicas se asocian a actividades fundamentales en la supervivencia y el desarrollo del ser humano ${ }^{41}$ :

El principio de las mecánicas de recolección reside en el acopio y aprovisionamiento de alimentos para garantizar la supervivencia. Siguiendo este principio de actuación, la búsqueda de objetos dentro del juego se identifica con la prevención o la lucha contra la enfermedad. De esta manera, los diferentes escudos que garantizan la protección contra los efectos secundarios de la quimioterapia son el objetivo en Ben's Game, mientras que la protagonista de $I$, Hope emprende una búsqueda de conocimiento y armas (gafas del conocimiento, guanteletes de fuerza, gong del coraje y cuerno del respaldo) que le permitan derrotar al monstruo que amenaza su aldea.

Las mecánicas de captura están vinculadas a "cazar a otros animales y enfrentarse a enemigos, eliminándolos o bien neutralizándolos”¹ . La articulación del combate con las diferentes manifestaciones de la afección plantea la lucha por la supervivencia del jugador frente a la enfermedad que se refleja, particularmente, en Re-Mission, ReMission 2, Ben's Game o en I, Hope. La propia construcción visual de las herramientas y armas para combatir la enfermedad pasa por elementos clásicos de los juegos de acción como el blaster médico multi-usos de Roxxi en Re-Mission y otras propuestas genéricas.

Las mecánicas de habilidad implican al jugador a través de retos acordes a la propuesta narrativa e integrados en una curva de aprendizaje y progreso para mantener su interés. Dichas mecánicas se adaptan al público al que va dirigido el juego. De esta forma, los requisitos de habilidad de AlphaBeatCancer propone unas mecánicas simples que contrastan con el mayor nivel de exigencia de otros juegos como I,Hope 
o, para públicos adultos, NanoDoc. Por último, tiene interés señalar su minimalismo en That Dragon, Cancer que refuerza el carácter testimonial y narrativo de este juego frente a sus homólogos (Figura 5).
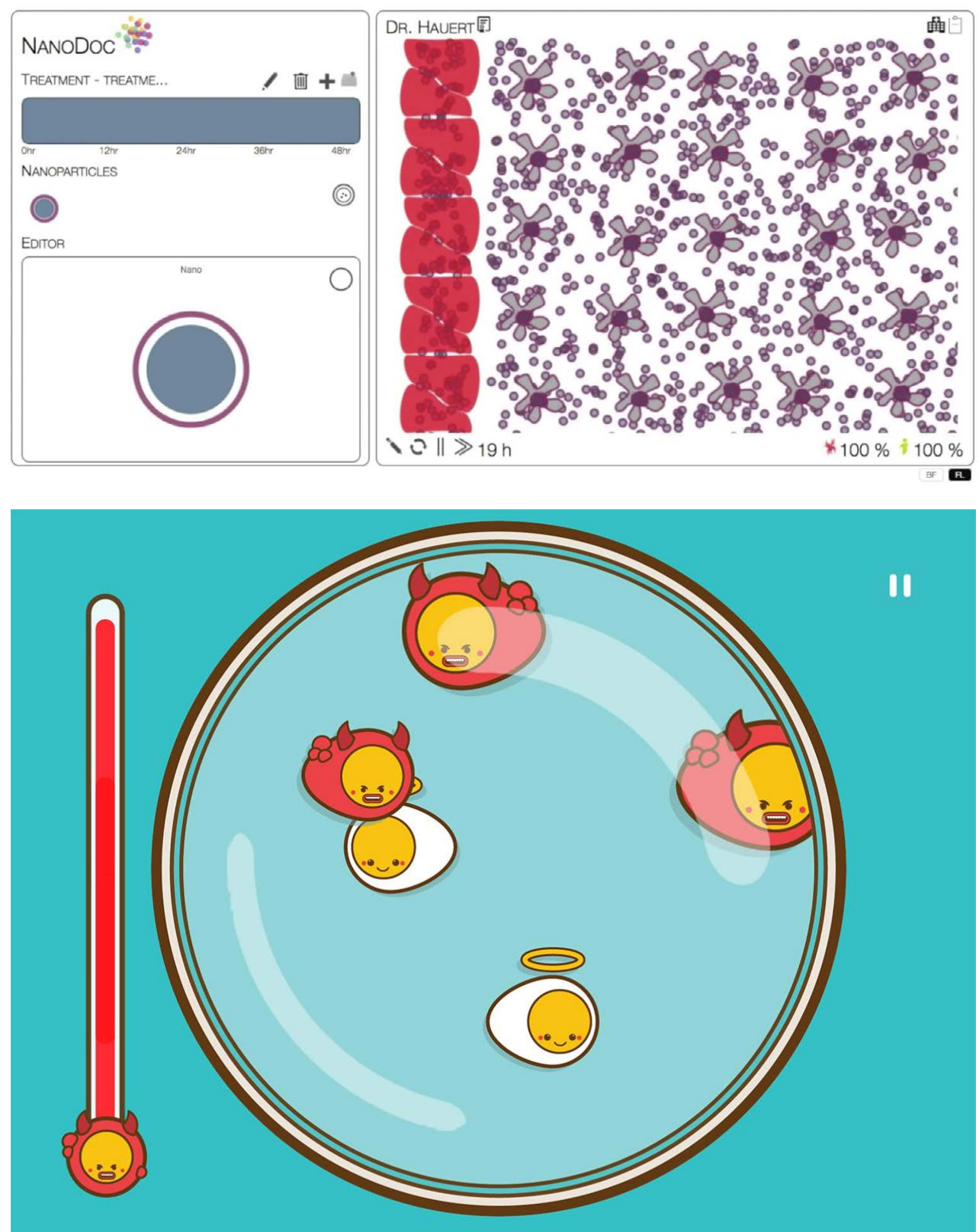

Figura 5. Mecánicas de habilidad de dificultad creciente.

Por último, las mecánicas de configuración - propias de los juegos de estrategia y de gestión de recursos- son menos habituales. Por ejemplo, en Re-Mission 2 se reducen a expresiones menores, aunque, sin embargo, son las mecánicas principales en el juego online NanoDoc. Dicho juego se ceñía a la resolución de puzles basados en patrones configuraciones reales de células tumorales- para que los usuarios ofrecieran soluciones que, posteriormente, fueron probadas con tumores reales ${ }^{42}$. 


\section{That Dragon, Cancer. el videojuego como testimonio}

That Dragon, Cancer ofrece una concepción experimental de su narrativa audiovisual e interactiva por su propósito conmemorativo. La acción del jugador se centra en la exploración del entorno virtual sin recibir apenas indicaciones sobre qué debe hacer. Por ejemplo, en la primera de ellas, el jugador controla a un pato que deambula por una charca y que puede encontrar unos trozos de pan que le lanza Joel, lo que permite escuchar la conversación entre los padres sobre la enfermedad del niño y las limitaciones que supone en su desarrollo cognitivo. A partir de ahí, el jugador va cambiando su identidad en las diferentes escenas (médico, padre, madre o un observador casual que distrae al niño en el hospital) para orquestar un conjunto de visiones sobre el sufrimiento del enfermo y su familia de forma cercana a los testimonios que se podrían encontrar en un documental. La construcción de esta experiencia virtual prima la inmersión y la recreación virtual sobre la rapidez o ejecución óptima durante el juego. El padre de Joel, desarrollador de That Dragon, Cancer, explicaba este propósito de la siguiente manera:

[...] Los videojuegos, a diferencia de otros medios, permiten persistir en los momentos... recordando el tiempo que pasamos con Joel, no se trata de lo que dijo o de los sucesos que recordamos, sino de esos espacios entre medias... Creo que los videojuegos permiten reposar/detenerse en ese espacio mejor que cualquier otra $\cos \mathrm{a}^{40}$.

Por último, el propósito documental de este videojuego permite reflejar las convicciones cristianas de sus creadores. En ese sentido, el juego expresa una dialéctica entre medicina y fe como forma de gestionar el dolor y aceptar situaciones dramáticas ${ }^{43}$. Un planteamiento reflejado en varias escenas donde el recurso a la oración alivia el sufrimiento del personaje y que plantea una dimensión espiritual exclusiva de esta propuesta. Una particularidad que expresa las formas en que la enfermedad y la muerte son interpretadas en las sociedades contemporáneas ${ }^{40}$.

\section{Desenlaces: perder una batalla no es perder la guerra}

La construcción de las condiciones que determinan el final de la experiencia de juego -y su lectura como victoria o derrota-contribuye al sentido del discurso. Los juegos de la muestra responden a una formulación clásica en torno a victorias por objetivos -eliminación de amenazas, completar un recorrido o resolver una serie de puzles- que se complementan con otros indicadores - puntos o logros secundarios-. La experiencia de juego se alinea con los propósitos emocionales asociados a los videojuegos: gestión de la rabia y la frustración, motivación, obtención de perspectiva, empoderamiento y relajación ${ }^{44}$. El interés por alcanzar estos objetivos explica la tenue dificultad de los juegos para mantener la atención de un público infantil o casual. Además, juegos como Re-Mission incorporan la activación de ventajas como la invulnerabilidad o el armamento ilimitado que, si bien rompen la lógica de desafío del videojuego para un jugador tradicional, permiten disfrutarlo de forma mucho más relajada y facilitar el éxito de sus objetivos discursivos. 
La victoria se asocia a derrotar a la representación del cáncer reforzando la propuesta de empoderamiento y una lógica de pensamiento positivo pretendida por los creadores: "[...]para obtener el conocimiento a través de la narración exploratoria, los jugadores son retados a obtener las llaves para una buena salud dentro de una narrativa creativa y realista" 36 . Las investigaciones clínicas empleando juegos como ReMission han señalado que esta experiencia suponía para los jugadores un incremento en la percepción de los riesgos asociados a la enfermedad y el interés por solicitar información relacionada con la misma ${ }^{45}$.

La otra cara de la moneda de cualquier juego es la derrota debida a la comprensión incompleta de sus mecanismos o el desempeño ineficiente de la habilidad del jugador frente a los retos que se le proponen. El aspecto más significativo de los juegos de la muestra es que refuerzan su propósito de empoderamiento minimizando las consecuencias negativas de los fallos de los jugadores y que parten de un respeto virtual a la vida y la muerte, hecho marcadamente paradójico en un medio que ha trivializado ambos conceptos ${ }^{40}$.

De este modo, hay un conjunto de juegos, entre los que se encuentran Cancer game y Nanodoc, en los que no existe posibilidad de fracaso, dado que en ningún momento se puede perder. Sólo se ofrece la posibilidad de avanzar a diferente velocidad y obtener una mayor o menor puntuación. De esta forma, al obviar la derrota, se redunda en el discurso positivo.

Los juegos que penalizan los errores de los jugadores plantean un proceso activo de aprendizaje. Se alienta al jugador y se le ofrece una nueva oportunidad de lograr la victoria Las formas de conseguirlo parten de la desactivación temporal del nanobot en Re-Mission -como un mero contratiempo-, pasando por concepciones más sencillas como el "Inténtalo de nuevo" en Ben's Game y Re-Mission 2, o la enunciación más eufemística de I, Hope, en la que el jugador retoma lo que estaba haciendo unos segundos antes del fracaso, planteando una narrativa alternativa con un lacónico "Así no es como va la historia”.

Por último, That Dragon, Cancer vuelve a diferenciarse del resto de propuestas por la ausencia de victoria y la omisión de cualquier tipo de indicador de progresión más allá de lo estrictamente narrativo. En ese sentido, el propósito del juego es “permitir al jugador contemplar y reconocer/admitir las pérdidas y ganancias que presenta el juego" ${ }^{40} \mathrm{y}$ reflejado en la idea de que: "[...] Nuestro viaje se ha caracterizado por la esperanza y muchos pequeños milagros, una comunidad de fe y un conjunto de médicos increíbles. E incluso, en el caso de que lo perdamos [a Joel], nuestro deseo es que la esperanza permanezca" ${ }^{\prime 4}$.

\section{Discusión y conclusiones}

La tradición académica que inscribe el juego como estrategia cognitiva del ser humano para explicar y entender el mundo ${ }^{47}$ ha enmarcado esta investigación sobre la contribución de los videojuegos al entramado simbólico que la sociedad otorga al cáncer. Estos videojuegos ofrecen alguna de las respuestas posibles desde ángulos diferentes: el empoderamiento, el testimonio, la medicina o la inculcación de hábitos preventivos ${ }^{43}$. Por tanto, comparten la percepción de que el medio puede ofrecer 
algo más que entretenimiento en un tema de este calado. Un rasgo que se confirma en los objetivos enunciados por los desarrolladores, el diseño de los videojuegos, la participación de instituciones o su relevancia mediática.

Esta visión de los creadores refleja su convicción del potencial del medio por el interés que despierta en colectivos juveniles, su rejugabilidad, que permite una consolidación del mensaje, y su potencial de inmersión o el aspecto cooperativo de muchos de estos juegos, que permite establecer espacios de diálogo sobre aspectos complejos de la enfermedad. En definitiva, se plantea un interés por obtener y sumarse a los "resultados [que] sugieren que un videojuego cuidadosamente diseñado puede tener un impacto positivo sobre los hábitos de salud en los jóvenes aquejados con enfermedades crónicas" 35 .

Las propuestas narrativas y lúdicas de los videojuegos proponen una visión del cáncer que permite su recodificación cultural por parte de los jugadores, independientemente de que su origen provenga de su propia experiencia o de la construcción mediada. En ese sentido ha destacado la adaptación narrativa a través de una construcción épica de combate o de solución de puzles que fomenta la motivación extrínseca en lo relacionado con la enfermedad o los tratamientos a seguir. El respaldo de otras propuestas narrativas (That Dragon, Cancer) tiene que ver tanto con su temática como con su originalidad para explorar una lógica documental del medio y una dimensión espiritual sobre la enfermedad. En definitiva, el potencial de los videojuegos para establecer una reflexión o una mediación sobre experiencias complejas.

La capacidad interactiva del videojuego favorece la atención en torno al discurso porque exige acciones inmediatas y refuerza lecturas de empoderamiento al proponer soluciones inmediatas y concretas a la amenaza. En ese sentido, la simplificación de objetivos, reglas y mecanismos de juego junto a un diseño en positivo propone una experiencia vicaria de superación y exploración de las relaciones entre causa y efecto que identifica al usuario como el principal artífice de su curación. La capacidad inmersiva de los videojuegos reforzaría esta enunciación como medio privilegiado del ecosistema comunicativo natural de los nativos digitales.

En conclusión, el análisis presenta indicios interesantes sobre las formas de actuación de los videojuegos en procesos de empoderamiento, construcción de empatía, identificación y, en definitiva, del potencial del videojuego en contextos no vinculados con el mero entretenimiento o el escapismo. Sin embargo, conviene tener en cuenta que ningún juego de esta muestra ha obtenido las ventas de los títulos más populares y el interés que han generado proviene del carácter excepcional de estas propuestas en la industria de los videojuegos.

\section{Contribución de los autores}

Todos los autores participaron activamente en todas las etapas de elaboración del manuscrito. 


\section{Agradecimientos}

Este artículo es producto del Proyecto de Investigación titulado "Politainment en el entorno de la posverdad: nuevas narrativas, clickbait y gamificación”, referencia CSO2017-84472-R, financiado dentro del Plan Estatal de Investigación, Desarrollo e Innovación del Ministerio de Economía y Competitividad. Convocatoria 2017. Parte de esta investigación ha sido apoyada por el Ministerio de Educación, Cultura y Deporte del Gobierno de España a través de un contrato del Subprograma de Formación de Profesorado Universitario (FPU14/05297).

\section{Derechos de autor}

Este artículo está bajo la Licencia Internacional Creative Commons 4.0, tipo BY (https:// creativecommons.org/licenses/by/4.0/deed.es).

\section{Referências}

1. Organisation for Economic Co-operation and Development. Health at a Glance 2017: OECD Indicators. Paris: OECD Publishing; 2017. doi: https://dx.doi.org/10.1787/ health_glance-2017-en.

2. World Health Organization. Cáncer [Internet]. Genva: Who; 2018 [citado 15 Mar 2018]. Disponible en: http://www.who.int/es/news-room/fact-sheets/detail/cancer

3. Eliott J, Forster AJ, McDonough J, Bowd K, Crabb S. An examination of Australian newspaper coverage of the link between alcohol and cancer 2005 to 2013. BMC Public Health. 2017; 18(1):47. doi: https://dx.doi.org/10.1186/s12889-017-4569-0.

4. Jensen JD, Moriarty CM, Hurley RJ, Stryker JE. Making sense of cancer news coverage trends: a comparison of three comprehensive content analyses. J Health Commun. 2010; 15:136-51. doi: https://dx.doi.org/10.1080/10810730903528025.

5. Icart-Iserna MT, Rozas-García MR, Sanfeliu-Cortes V, Viñas-Llebota H, FernándezOrtega MP, Icart MC. El cáncer en el cine. Un recurso para los profesionales de la salud. Educ Med. 2009; 12(4):239-46.

6. Abella A. Cómics contra los tabús del cáncer. elPeriódico [Internet]. 2017 [citado 16 Mar 2018]. Disponible en: https://www.elperiodico.com/es/ocio-y-cultura/20170203/ cancer-comic-santalo-castro-hayden-hernandez-5779361

7. The Pop Mythologist. 10 comic book superhéroes who battled cáncer. Pop Mythology [Internet]. 2016 [citado 16 Mar 2018]. Disponible en: https://www.popmythology. com/superheroes-who-battled-cancer/

8. Wen KY, McTavish F, Kreps G, Wise M, Gustafson D. From diagnosis to death: a case study of coping with breast cancer as seen through online discussion group messages. J Comput Mediat Commun. 2011; 16(2):331-61. doi: https://dx.doi.org/10.1111/ j.1083-6101.2011.01542.x.

9. Fernández-Gómez E, Díaz-Campo J. Comunicación sobre el cáncer en facebook: las asociaciones de Argentina, Chile, Colombia y España. Cuad Info. 2016; (38):35-50. doi: https://dx.doi.org/10.7764/cdi.38.926. 
10. Chou WYS, Hunt Y, Folkers A, Augustson E. Cancer survivorship in the age of youtube and social media: a narrative analysis. J Med Internet Res. 2011; 13(1):e7. doi: https://dx.doi.org/10.2196/jmir.1569.

11. Cegarra J. Fundamentos teórico epistemológicos de los imaginarios sociales. Cinta Moebio. 2012; 43:1-13.

12. Castoriadis C. La institución imaginaria de la sociedad. Buenos Aires: Tusquest; 2007.

13. Pinto JL. Los imaginarios sociales del delito: la construcción social del delito a través de las películas (1930-1999). Anthropos. 2003; 198:161-76.

14. Song M, Vogelstein B, Giovannucci EL, Willett WC, Tomasetti C. Cancer prevention: molecular and epidemiologic consensus. Science. 2018; 361(6409):1317-8. doi: https://dx.doi.org/10.1126/science.aau3830.

15. Brandon M. Targeted social media initiatives for cancer prevention in young adults. Oncol Times. 2018; 40(19):18-9. doi: https://dx.doi.org/10.1097/01. COT.0000547233.25805.cc.

16. Wilkinson S, Kitzinger C. Thinking differently about thinking positive: a discursive approach to cancer patients' talk. Soc Sci Med. 2000; 50(6):797-811. doi: https:// dx.doi.org/10.1016/S0277-9536(99)00337-8.

17. Wakefiled MA, Loken B, Hornik RC. Use of mass media campaigns to change health behaviour. Lancet. 2010; 376(9748):9-15. doi: https://dx.doi.org/10.1016/S01406736(10)60809-4.

18. Sontag S. La enfermedad y sus metáforas. El sida y sus metáforas. Madrid: Taurus; 2003.

19. Gibbs Jr. RW, Franks H. Embodied metaphor in women's narratives about their experiences with cancer. Health Commun. 2002; 14(2):139-65.

20. Camus JTW. Metaphors of cancer in scientific popularization articles in the British press. Discourse Stud. 2009; 11(4):465-95. doi: https://dx.doi. org/10.1177/1461445609105220.

21. Hauser DJ, Schwarz N. The war on prevention: bellicose cancer metaphors hurt (some) prevention intentions. Pers Soc Psychol Bull. 2015; 41(1):66-77. doi: https:// dx.doi.org/10.1177/0146167214557006.

22. Hanahan D. Rethinking the war on cancer. Lancet. 2014; 383(9916):558-63. doi: https://dx.doi.org/10.1016/S0140-6736(13)62226-6.

23. Kleinke AM, Classen CF. Adolescent and young adults with cancer: aspects of adherence - a questionnaire study. Adolesc Health Med Ther. 2018; 9:77-85. doi: https://dx.doi.org/10.2147/AHMT.S159623.

24. Sudhakar A. History of cancer, ancient and modern treatment methods. J Cancer Sci Ther. 2009; 1(2):1-4. doi: https://dx.doi.org/10.4172/1948-5956.100000e2.

25. Kastbom L, Milberg A, Karlsson M. A good death from the perspective of palliative cancer patients. Support Care Cancer. 2017; 25(3):933-9. doi: https://dx.doi. org/10.1007/s00520-016-3483-9.

26. Gómez S, Gil A, Carrillo JA, Navarro N. Constructing Donald Trump: mobile apps in the political discourse about the president of the United States. Comunicar. 2019; 59. doi: https://dx.doi.org/10.3916/C59-2019-05. 
27. Castillo D. Cancer research UK launches second advergame with Matmi to promote run 10K events in 2010 [Internet]. 2010 [citado 15 Mar 2018]. Disponible en: http:// productplacement.biz/201006252584/branded-entertainment/cancer-research-uklaunches-second-advergame-with-matmi-to-promote-run-10k-events-in-2010.html

28. Flanagan M, Nissenbaum H. Values at play in digital games. Cambridge: MIT Press; 2014.

29. Muriel D, Crawford G. Video games as culture: considering the role and importance of video games in contemporary society. London: Routledge; 2018.

30. Navarro-Remesal V. Libertad dirigida: una gramática del análisis y diseño de los videojuegos. Santander: Shangrila; 2016.

31. Planells AJ. Videojuegos y mundos de ficción: de Super Mario a Portal. Madrid: Ediciones Cátedra; 2015.

32. Pérez-Latorre $\mathrm{O}$, Oliva $\mathrm{M}$, Besalú R. Videogame analysis: a social-semiotic approach. J Soc Semiotics. 2016; 27(5):586-603. doi: https://dx.doi.org/10.1080/10350330.2016 .1191146 .

33. Stacey J. Teratologies: a cultural study of cancer. London, New York: Routledge; 1997.

34. Make-A-Wish America. Ben's video game fights cancer [Internet]. Phoenix: Make-AWish America; 2007 [citado 15 Mar 2018]. Disponible en: http://wish.org/wishes/ wish-stories/i-wish-to-be/ben-video-game-creator\#sm.00002hv52b3zncrospv2lzispjxjj

35. Kato PM, Cole SW, Bradlyn AS, Pollock BH. A video game improves behavioral outcomes in adolescents and young adults with cancer: a randomized trial. Pediatrics. $2008 ; 122(2): \mathrm{e} 305-17$.

36. Wei W. Cancer game: interactive narratives through explorational storytelling [Internet]. 2007 [citado 18 Mar 2018]. Disponible en: http://veevia.com/project/ Cancer_Game/index.html

37. NanoDoc [Internet]. 2013 [citado 18 Mar 2018]. Disponible en: https://scistarter. com/project/853-NanoDoc

38. Wolf MJP. Genre and the video game. In: Raessens J, Goldstein F, editores. Handbook of computer game studies. Cambridge: MIT Press; 2005. p. 193-204.

39. Bloom BS. Daily regimen and compliance with treatment: fewer daily doses and drugs with fewer side effects improve compliance. BMJ. 2001; 323(7314):647. doi: https:// dx.doi.org/10.1136/bmj.323.7314.647.

40. Schott GR. That dragon, cancer: contemplating life and death in a medium that has frequently trivialized both. In: Proceedings of the Digital Games Research Association International Conference; 2017; Melbourne, Australia. Melbourne: DiGRA; 2017. v.14, n.1, p. 1-10.

41. Pérez Ó. From chess to starcraft. A comparative analysis of traditional games and videogames. Comunicar. 2012; 38:121-9. doi: https://dx.doi.org/10.3916/C38-201203-03.

42. Hodson H. Gamers unleash swarms of nanoparticles on tumours [Internet]. 2013 [citado 18 Mar 2018]. Disponible en: https://www.newscientist.com/article/ mg21929344.600-gamers-unleash-swarms-of-nanoparticles-on-tumours/

43. Auxier JW. That dragon cancer goes to seminary: using a serious video game in pastoral training. Christian Educ J. 2018; 15(1):105-17. doi: https://dx.doi. org/10.1177/0739891318759725. 
44. De la Hera $\mathrm{T}$. The persuasive roles of digital games: the case of cancer games. Media Commun. 2018; 6(2):103-11. doi: https://dx.doi.org/10.17645/mac.v6i2.1336.

45. Khalil GE. When losing means winning: the impact of conflict in a digital game on young adults' intentions to get protected from cancer. Games Health J. 2012; 1(4):279-86. doi: https://dx.doi.org/10.1089/g4h.2012.0017.

46. Green R. Why we're making that dragon, cancer [Internet]. 2013 [citado $18 \mathrm{Mar}$ 2018]. Disponible en: http://www.thatdragoncancer.com/thatdragoncancer/whywere-making-that-dragon-cancer

47. Frasca G. Jugar, videojuegos y creación de sentido. Una introducción. Comunicación. 2009; 7(1):37-44.

This article analyzes the intersection between cancer - one of the leading causes of death in contemporary society - and one of the most important forms of media in twentieth-century popular culture: video games. An analysis was conducted of messages conveyed by eight videogames launched in the last 15 years with narratives on the disease, patients, and cancer treatment and outcomes. The findings point to a narrative construction that depicts the most common features of the disease, focusing on social empowerment through the use of science, social support, and spiritual convictions.

Keywords: Cancer. Video games. Serious games. Cultural industries. Information and awareness raising.

Neste artigo analisamos a interseção entre o câncer - uma das principais causas de mortalidade nas sociedades contemporâneas - e uma das mídias mais significativas da cultura popular no século 21: os videogames. A análise de conteúdo das mensagens foi composta por oito videogames que, nos últimos 15 anos, revelam uma abordagem narrativa em torno da condição dos pacientes, dos tratamentos e do desfecho da doença. Os resultados apontam para uma construção em que permanecem seus traços mais populares, voltados para o empoderamento social frente à doença, por meio do uso da ciência, apoio social ou das convicçóes espirituais.

Palavras-chave: Câncer. Videogame. Jogos sérios. Indústrias culturais. Informação e conscientização. 